\title{
A Conceptual Mirror: Towards a Reflectional Symmetrical Relation between Mentor and Learner
}

\author{
Farshad Badie
}

\begin{abstract}
The multilevel interactions between a mentor and her/his learner could exchange various conceptions between them that are supported by their own conceptualisations. Producing the own realisation of a world and developing it in the context of interactions could be said to be the most valuable product of the constructivist interactions. The most significant matter in meaning construction is producing the own meaningful comprehension, realisation and understanding. Here the learner gets to know how to develop her/his thinking. In this research, I will focus on relating (i) meaning construction through the lenses of the learner's conceptions and (ii) meaning construction through the lenses of constructivism. Constructivism is an educational theory of learning and a model of knowing. The main contribution of this research is analysing the symmetrical relationship between learner and mentor. I will analyse the logical dependencies between learner and mentor and will check their reflectional symmetrical relationship in a conceptual mirror. The conceptual mirror is a phenomenon that represents the meeting point of the mentor's and the learner's conceptual knowledge.
\end{abstract}

Index Terms-Conceptual knowledge, conceptual mirror, constructivism, interaction, meaning construction.

\section{INTRODUCTION AND MOTIVATION}

In an interaction between a mentor and her/his learner a number of questions, answers, actions and reactions concerning their personal conceptions are exchanged. First, I shall emphasise that what I use and express under the label of a 'concept' aims at providing a comprehensible characteristics of conceptions and conceptualisations. In my approach, a concept is a linkage between linguistic expressions and the mental images (in a broad sense) that the learner (mentor) may have in her/his mind, see [1]. For instance, these mental images could be interpreted and seen as the learner's representation of aspects of the world (of the universe of discourse). Also, the mentor's construction of the universe of discourse is another instance of mental images. According to the features of concepts just mentioned, a learner's (mentor's) conception within an interaction is equivalent to her/his act of imaging various concepts and linking her/his expressions with regard to the own mental images and schemata ${ }^{1}$. In my approach, a learner's (mentor's) schemata i) provide backgrounds for her/his concepts, ii) specify her/his inferences and reasonings, iii) describe various

Manuscript received October 10, 2015; revised January 7, 2016.

Farshad Badie is with Aalborg University, Rendsburggade 14, 9000 Aalborg, Denmark (e-mail: badie@id.aau.dk).

${ }^{1}$ http://plato.stanford.edu/entries/schema-http://www.britannica.com/top ic/schema-cognitive theories based on terminologies and world descriptions, and finally iv) give sufficient and satisfying conditions for definitions of truth.

The multilevel, and commonly agreement-oriented, interactions between a mentor and her/his learner could be viewed as the radical constructivist account of the learner's (and the mentor's) realisation and comprehension, see [2] for more details. The constructivist account of an agent's realisation is capable of enabling her/him in developing the individual realisations of the concepts. Producing the own realisation (and thus, understanding) of a world and developing it during the interaction with the interlocutor could be said to be the most valuable product of the constructivist interactions. In a constructivist interaction learner and mentor develop their own realisations of the underlying systematic processes in reality, and also their realisations of themselves through the universe of discourse. Constructivism is a model of knowing and is an educational theory of learning. It conceives of learning as the process of construction. In the framework of constructivism a learner attempts to construct knowledge based upon her/his preconceptions (pre-concept formations ${ }^{2}$ ) and pre-structured knowledge. The main focus of the mentor could be said to be on the learner's knowledge construction. Consequently, the learner will have the opportunity to attain deeper personal realisations and greater motivations, see [2], [3]-[7]. A learner, either by acquiring new concepts or by modifying existing concepts, decides to construct knowledge. And the mentor constructs parts of the learner's mind by performing the constructive mentoring methods and theories. Actually, what a learner constructs could be analysed as the reflection of what the mentor has provided for her/him (e.g., asked her/him a question). Also, what a mentor constructs in the learner's mind could be seen as be the reflection of what the learner has done (e.g., answered a question to the mentor).

In this research I see learning from the functional point of view and think of causation in the process of construction. In my opinion, knowledge can actively be constructed based upon the learner's realisation of the meanings of various concepts with regard to their descriptions and definitions. I have focused on this area in [8], [9]. Kindly observe that the definition of a concept is an equation whose left-hand side is a concept and whose right-hand side is a description for that concept, see [10]. Also, a meaning is a context-update function, see [11]. Additionally, I have defined a meaning as a concept-update function in my approach, see [9].

Focusing on the learners' conceptions of successful learning and effective mentoring, a learner can describe the

\footnotetext{
${ }^{2}$ http://teachinghistory.org/teaching-materials/teaching-guides/25184
} 
steps of learning from two distinct points of view in order to provide a satisfactory description of knowledge construction development. The first one is her/his own point of view and the second one is her/his mentor's point of view. Learners usually observe learning through the lenses of their mentors in order to see themselves and their own requirements. Additionally, a learner needs to realise and to figure out how her/his own conceptions of learning about an object may be reflected in the mentor's conceptions of that object and vice versa.

Now I take a model of students' developing conceptions of learning into consideration. The model sketches on Säljö's seminal studies on learning conceptions, see [10]. He focused especially on describing learning from the learner's point of view and identified five categories and levels for a learner's developing conceptions of learning. Also, [11] suggested a new category and added this category as the sixth level to the Säljö's model. Here I summarise the model as the following items:

- Knowing More. The learner observes learning as knowing new things. S(he) wants her/his mentor to impart the well-structured information into separated and isolated facts.

- Memorising. The learner reproduces what $\mathrm{s}(\mathrm{he})$ has acquired and known. So, it's all about memorising. S(he) still tries to know more in order to reproduce more.

- Selection. The learner selects and memorises the facts and might be able to apply her/his knowledge in practical approaches. $\mathrm{S}(\mathrm{he})$ expects the mentor to shape and to motivate her/him during the interaction.

- Meaning Construction. The learner is realising that constructing knowledge is very important and it will be plausible in the shadow of meaning construction. The mentor would guide the learner to find out how to think logically, analytically and productive.

- Reality Interpretation. Learning as an interpretative process should support the learner in interpreting and understanding reality. Then the learner characterises learning as the process of self development.

- Self Awareness (Self Realism). This category is about self realisation. This process is always going to be continued. The learner is always going to expand her(him)self. Obviously, this is the most excellent and the most transcendental conception.

In this article my main focus is on Meaning Construction (level four). I have been focusing on meaning construction in the context of interactions and have written some of my research products in [8], [9]. In my opinion, this level is the most definitive level. Let me make a conceptual linkage between my own approach and Säljö's model. Focusing on meaning construction, the learner deals with her/his individual concept constructions for developing conceptualisations. So I shall bring your attention to the fact that my approach recognises the collection \{Concept Formation, Concept Transformation, Concept Reformation $\}$ as the most significant matter in the development of concept constructions within constructivist interactions. I have identified the process 'Concept Formation $\rightarrow$ Concept Transformation $\rightarrow$ Concept Reformation' as the process of Concept Construction (CC) in the context of interaction. The most significant expressions at this level are 'meaningful comprehension', 'realisation' and 'understanding'. Here the learner gets to know (and gets to identify) how to relate different ideas. In fact, $s($ he) is about to develop her/his thinking. As mentioned, in my opinion, knowledge can actively be constructed based upon the learner's realisation of the meanings of various concepts with regard to their descriptions and definitions. So, meaning construction in the framework of constructivism and in the context of interactions (and dialogues) between the learner and the mentor finds its real significance here. Subsequently, the learner describes her/his individual concepts and attempts to produce meanings, to formulate them and to develop their constructions. At this level, the compassionate mentor is the developer of the learner's thinking. This development will support the learner in finding how to think logically, analytically and productive.

The main focus of this research is on a reflectional symmetrical relation between learner and mentor. I will logically analyse it while I will focus on conceptual knowledge. Thus I need to analyse the logical dependencies between learner and mentor, and see the reflections in a conceptual mirror. The conceptual mirror represents the meeting point of the mentor's and the learner's conceptual knowledge. In the following sections I will present the following: The Learner's Conceptual Knowledge, The Relationships Between Learner And Mentor, Conceptual Mirror: A Reflection Symmetry and Conclusions.

\section{THE LEARNER's CONCEPTUAL KNOWLEDGE}

Bloom's taxonomy ${ }^{3}$ is a framework for classifying pedagogical objectives, which could be interpreted as the statements of what educators and mentors expect their learners to have learned, see [12], [13]. According to Bloom's researches, knowledge has a strong relationship with recognition of various materials, ideas, methods, processes, structures and settings. Bloom's taxonomy divides a knowledge class into multiple classes (e.g., knowledge of terminologies, knowledge of ways and means, knowledge of trends and sequences, knowledge of classifications and categorisations, knowledge of criteria, knowledge of methodologies, knowledge of quantifications, knowledge of principles - generalisations and specifications, knowledge of theories and structures). Since then, [13] has proposed a knowledge dimension in the revised version of Bloom's taxonomy. The revised taxonomy consists of Factual Knowledge (e.g., terminological knowledge), Conceptual Knowledge (e.g., knowledge of theories, models and structures), Procedural Knowledge (e.g., knowledge of methods and algorithms) and Metacognitive Knowledge (e.g., contextual knowledge, conditional knowledge).

In fact learning consists of a sort of transformation functions from knowledge (that is going to be known) into the sets of 'facts', 'procedures' and 'concepts' in different 'contexts'. And subsequently, the learners transform facts, procedures and concepts into their minds. I formally describe learning as the conjunction of the following transformations:

\footnotetext{
3 www.icels-educators-for-learning.ca
} 
Knowledge $\rightarrow\{$ Fact , Procedure , Concept $\}$

$\{$ Fact, Procedure, Concept $\} \rightarrow$ Mind .

In this research my main concern is 'concepts'. I am focusing on conceptual knowledge acquisition. In myopinion, there is a concept behind every fact. Then any factual knowledge can be supported by a conceptual knowledge. For instance, according to a fundamental characteristic of terminological knowledge (as a type of factual knowledge), we can represent terminologies by the means of taxonomies. A taxonomy could be constructed based upon concepts. Then a terminological knowledge has been supported by a conceptual knowledge. Also, as another instance, we can define a body of the related elements and interpret it as a set of constructors for denoting various concepts and their interrelationships. That's how the concept languages and descriptive languages appear. Then, we could be able to represent knowledge over concepts, their instances and their relationships. Additionally, in my opinion, any procedure could be observed as the conclusion of the sequence of a number of facts. Therefore:

Fact $\rightarrow$ Fact $\rightarrow$ Fact ... $\Leftrightarrow$ Procedure.

And actually:

1) A procedure could be viewed as a body of a few number of facts.

2) A fact is supported by a concept.

3) A procedure is supported by a concept.

According to the afore-mentioned items, a learner acquires facts and procedures and they all get supported by concepts in her/his mind. These concepts are considered as the building blocks of her/his conceptual knowledge and can be considered as the elements and ingredients of a conceptual system, and thus support the learners' developing conceptualisation of learning. Then, s(he) can think of learning (mentoring), successful learning and satisfactory mentoring. Here I describe learning as the conjunction of the processes i) and ii):

i) Knowledge $\rightarrow$ \{Fact, Procedure, Concept $\} \rightarrow$ \{Concept, Concept, Concept $\}$

ii) $\{$ Concept, Concept, Concept $\} \rightarrow\{$ Fact, Procedure, Concept $\} \rightarrow$ Mind.

\section{THE RELATIONSHIPS BETWEEN LEARNER AND MENTOR}

In logic the reflexive relation $R$ is a binary relation between an object and itself. Let $R$ be a relationship on set $A$. If and only if $R$ relates every element of $A$ with itself, then $R$ is identified as a reflexive relation on $A$. Formally, $\forall a_{i} \in A, a_{i}$ $R a_{i}$. A binary relation $R$ between two elements of set $A$ is identified as a symmetrical relation if, and only if, for any $a$. and $a_{j}$ belonging to $A$, in the case $R$ relates $a_{i}$ with $a_{j}$, then $R$ relates $a_{j}$ with $a_{i}$ as well. Formally, $\forall a_{i} \in A \exists a_{j} \in A ; a_{i} R$ $a_{j} \Rightarrow a_{j} R a_{i}$. Let set $E$ be a learning environment. A lot of elements could exist in a learning environment. The main focus of this research is on elements $L$ and $M$, where $L$ and $M$ denote Learner and Mentor. Formally, $L \in E$ and $M \in E$. $M$ can metaphorically be seen as a mirror that shows the $L$ 's self, see [14]. In my opinion, the multi-level agreement oriented interactions between a mentor and her/his learner constructs a symmetrical relationship between them. Let me conclude that a real constructive and productive relationship between $L$ and $M$ is inherently a symmetrical relationship. It may not be symmetric (and may be asymmetric) in some existing relationships over the set $E$. But, it is potentially a symmetry and may represent a willingness to achieve more symmetrical properties and preserve them in the context of interactions. A responsible learner, in parallel with her/his constructive and compassionate mentor, attempts to survive this symmetrical relationship. I see this characteristic as the most excellent and valuable realisation of learning phenomenon in the context of relationships between mentor and learner. Moreover, any person is able to observe the reflection of her/his own 'conceptions of learning phenomenon with regard to perceived facts, procedures and concepts', in her/his self (e.g., individuality, personality). In fact, this is also the most excellent and transcendental realisation of learning phenomena. Similarly, 'growing self awareness' is the most valuable product of the complement model of Säljö's model and has been manifested in the learner's self. Thus, there exists a reflexive relationship between 'growing self awareness' and 'self'. For another instance, the 'reality interpretation' is the product of the last level of Säljö's model. I assume that reality interpretation is also reflected in $L$ 's (and $M$ 's) interpretations and these interpretations could be made in the shadow of the learner's (and mentor's) self. Further, 'meaning construction' as the product of layer four of Säljö's model has been reflected in personal understanding based on individual interpretations in the shadow of the learner's (mentor's) self.

\section{CONCEPTUAL MIRROR: A REFLECTION SYMMETRY}

I will define the conceptual mirror in order to clarify the interrelationships between the learner's and the mentor's conceptual knowledge. A conceptual mirror can be a supportive point for mentor and learner. In fact, a mentor could have a better understanding of her/his learner's knowledge by looking in the mirror and the learner can have a better realisation of mentoring knowledge by looking in the mirror. I define the conceptual mirror on the meeting point of:

1) The learner's learning.

2) The reflections of the learner's conceptions in her(him)self. Similarly, the conceptual mirror is located on the meeting point of:

3) The mentor's mentoring.

4) The reflections of the mentor's conceptions in her(him)self.

I shall emphasise that the learner's trust is reflected in the learner's self. Thus, the learner's trust can be seen in the conceptual mirror at the meeting point of (B) and (D).

Logical Analysis of the Conceptual Mirror. There is a symmetrical relationship between Learner $L$ and Mentor $M$ that is represented by $c$ in Fig. 1. The symmetrical relationship $c$ could be described as the product of the concatenation of two reflexive relationships. These reflexive relationships are represented by $a$ and $b$ in Fig. 1. Let me clarify what this 
concatenation is. In this system the learner sees the reflection of her/his individual conceptions of learning phenomena in the conceptual mirror. S(he) also observes the reflection of the mentor's mentoring phenomena in the conceptual mirror, and subsequently, in her(him)self [Result 1]. On the other hand, the mentor has seen the reflection of her/his mentoring phenomena in the conceptual mirror. Moreover, the mentor observes the reflection of the learner's conceptions of learning phenomena in the conceptual mirror, and thus, $\mathrm{s}(\mathrm{he})$ visions the reflection of her/his mentoring phenomena in the learner's self [Result 2]. As for the results 1 and 2, the learner observes the reflection of the mentor's mentoring phenomena in her(him)self and the mentor visions the reflection of her/his mentoring phenomena in the learner's self. This demonstrates a symmetrical relationship between a mentor and a learner. So, we have seen that the concatenation of the reflexive relation $\boldsymbol{a}$ and reflexive relation $\boldsymbol{b}$ could produce the symmetrical relationship $c$. Let $R_{r}$ and $R_{s}$ denote the reflexive and symmetrical relationships. Therefore I have the following system:

$$
\begin{aligned}
& L R_{r} L \\
& M R_{r} M \\
& \left(\ldots R_{r \ldots}\right)^{R_{s}\left(\ldots R_{r} \ldots\right)}
\end{aligned}
$$

The first premise represents the reflexive relation between learner and her(him)self. Also, the second premise represents the reflexive relation between the mentor and her(him)self. The third premise represents that there is a symmetrical relation between two reflexive relations. Therefore, I conclude that there is a symmetrical relation between 'the reflexive relation between learner and her(him)self' and 'the reflexive relation between mentor and her(him)self'. Formally:

$$
L R_{r} L \quad R_{S} \quad M R_{r} M
$$

As described, the learner and mentor vision the relationship of their interlocutors (mentor and learner) with themselves in the conceptual mirror. In fact, the symmetrical relationship " $L$ $R_{r} L R_{s} M R_{r} M$ " enters the conceptual mirror. Reconsidering Section II, by learning a learner transforms knowledge into multiple concepts. Regarding the reflexive relationship between a learner and her(him)self in a learning process I conclude that her/his own conceptual knowledge (and produced concepts) reflects in her(him)self. Considering Lcas a learned concept (a produced concept based on learning), and taking the result (I) into account, I propose the following system:

$$
\begin{gathered}
L_{c} R_{r} L_{c} \\
M_{c} R_{r} M_{c} \\
\left(\ldots R_{r} \ldots\right) R_{s}\left(\ldots R_{r \ldots}\right)
\end{gathered}
$$

The first premise represents the reflexive relation between a learned concept and itself. The second one represents the reflexive relation between a mentored concept and itself. Also, the third premise represents that there is a symmetrical relation between two reflexive relations. So, there is a symmetrical relation between a 'reflexive relation between a learned concept and itself' and 'reflexive relation between a mentored concept and itself'. Then formally:

$$
L_{c} R_{r} L_{c} \quad R_{S} \quad M_{c} R_{r} M_{c}
$$

Moreover, this conclusion denotes that the learner observes the reflection of the mentor's conceptual knowledge in the conceptual mirror, where $\mathrm{s}(\mathrm{he})$ has observed the reflection of her/his conceptual knowledge. Therefore:

$$
L_{c} R_{r} L_{c} \Leftrightarrow M_{c} R_{r} M_{c} \text {. }
$$

It can be divided into two conclusions:

$$
\begin{gathered}
L c \operatorname{Rr} L c \Rightarrow M c \operatorname{Rr} M c \\
M c \operatorname{Rr} M c \Rightarrow L c \operatorname{Rr} L c .
\end{gathered}
$$

According to (i), the learner observes the learned concept in her(him)self. This concludes that the mentor observes the mentored concept in her(him)self. According to (ii), the mentor observes the mentored concept in her(him)self, and therefore, the learner observes the learned concept in her(him)self. These conclusions demonstrate an equivalence and stability between learner's and mentor's conceptual knowledge.

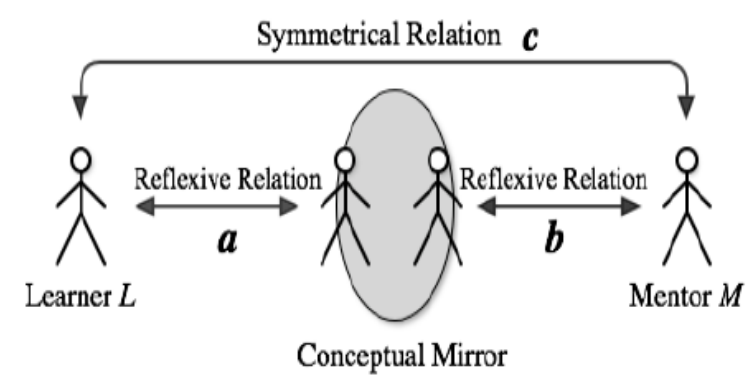

Fig. 1. Conceptual mirror.

\section{SUMMARY AND CONCLUSIONS}

An interaction between a mentor and her/his learner could exchange their personal conceptions. The multilevel interactions between them could be viewed as the radical constructivist accounts of their realisations and comprehensions. Producing one's own realisation of the world and developing it in the context of interaction could be said to be the most valuable product of the constructivist interactions. In this research, I have employed Säljö's model of students' developing conceptions of learning and have focused on one of its levels, so-called Meaning Construction. The main reasons for this consideration have been my special interest in meaning construction in the context of interactions and my own research in the analysis of meaning construction through the lenses of the theory and philosophy of constructivism. I believe that this level is the most definitive and determinative level in Säljö's model of students' 
developing conceptions of learning. In my opinion, this level can appropriately describe the interrelationship between the learners' and the mentors' observations. And, in fact, at this level, the learner initiates the developing of developing her/his conceptualisations. The most significant matter at this level is the production of the own meaningful comprehension and understanding. At this point the learner gets to know (and to identify) how to relate different ideas and how to develop her/his thinking. In this article I have made a conceptual linkage between my own approaches and Säljö's model. I have focused on the conceptual knowledge in the revised Bloom taxonomy with regard to my goals. The main contribution of this research has been logical representations and the analysis of the reflectional symmetrical relation between learner and mentor. Thus I have analysed the logical dependencies between learner and mentor, and have checked their reflections in a conceptual mirror. A conceptual mirror is a phenomenon that represents the meeting point of the 'learner's learning' and 'the reflections of the learner's conceptions in her(him)self'. It also represents the meeting point of the 'mentor's mentoring' and 'the reflections of the mentor's conceptions in her(him)self'. Accordingly, a conceptual mirror represents the junction (and dependency) of the mentor's and the learner's conceptual knowledge in the context of their interactions.

\section{REFERENCES}

[1] G. Hans, "Deviational syntactic structures," Bloomsbury Academic, London, 2013.

[2] S. Rand, F. Paul, J. Michael, and C. Richard, "Cognitive flexibility, constructivism, and hypertext: Random access instruction for advanced knowledge acquisition in ill-structured domains, random access instruction for advanced knowledge acquisition," Educational Technology, pp. 24-33, 1992.

[3] J. Piaget, "Six psychological studies," Random House, 1967.

[4] T. Husen and T. N. Postlethwaite, "Constructivism in education," The International Encyclopaedia of Education, vol. 1, Oxford, New York: Pergamon Press, 1989.

[5] B. McGaw and P. Peterson, Constructivism and Learning. International Encyclopaedia of Education, Oxford: Elsevier, 2007.

[6] S. R. Keith, "The cambridge handbook of the learning sciences," Cambridge Handbooks in Psychology, 2014.
[7] J. Barbara, Constructivism in Education, Hillsdale, NJ: Lawrence Erlbaum, 1995.

[8] B. Farshad, "A semantic basis for meaning construction in constructivist interactions," presented at CELDA 2015, 2015.

[9] B. Farshad, "Towards a semantics-based framework for meaning construction in constructivist interactions," in Proc. ICERI2015, pp 7995-8002, 2015.

[10] B. Franz, C. Diego, M. Deborah, N. Daniele, and P.-S. Peter, The DescriptionLogicHandbook: Theory, Implementation and Applications, Cambridge University Press, 2003.

[11] E. J. Rossum and S. M. Schenk, "The relationship between learning conception, study strategy and learning outcome," British Journal of Educational Psychology, 1984.

[12] M. D. Furst, E. J. Hill, W. H. Krathwohl, D. R. Bloom, and B. S. Engelhart, "Taxonomy of educational objectives: The classification of educational goals," Handbook I: Cognitive Domain, New York: David McKay Company, 1956

[13] R. K. David, “A revision of bloom's taxonomy: An overview," Theory into Practice, Routledge Publishers, 2002

[14] O. G. Gerald, "Teaching learners to be self-directed," Adult Education Quarterly, 1991

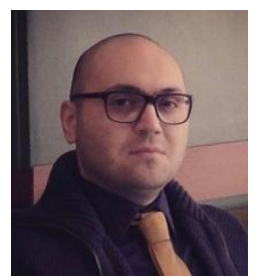

Farshad Badie was born in Tehran, Iran on May 14, 1984. He has been a $\mathrm{PhD}$ candidate in human centered communication and informatics doctoral program at the Doctoral School of the Humanities since October 2014. He is doing his $\mathrm{PhD}$ at Aalborg University, Aalborg, Denmark. His previous studies are: $\mathrm{PhD}$ internship in computer science (machine learning and ontology engineering), Institute for Applied Informatics, Department of Computer Science, University of Leipzig, Leipzig, Germany (2013-2014) - Master of Science (graded with honour) in software IT \& computer science, Informatics Faculty, University of Debrecen, Debrecen, Hungary. (2011-2013) - BSc in applied mathematics, Faculty of Basic Sciences, Tehran Central Branch University, Tehran, Iran. (2002-2008).

$\mathrm{He}$ is currently affiliated with the Center For Linguistics (CFL), Department of Communication, Aalborg University. CFL is located in Rendsburggade 14, 9000 Aalborg, Denmark. He will be the secretary of the Nordic Association of Linguists from January 2016. The previous academic positions are: research assistant at the Institute for Applied Informatics, Department of Computer Science, University of Leipzig, Germany; from 2013 to 2014, teaching assistant, seminars leader (Mathematical Logic in Computer Science), Department of Computer Science, University of Debrecen, Hungary from 2012 to 2013.

Mr. Badie is an executive committee member of Nordic Association of Linguists. He is also a member of Scandinavian Logic Society, Copenhagen Association for Dynamics Interaction Logic Language \& Computation, Association for Symbolic Logic and Nordic Society for Phenomenology. 